\title{
Re-inventing print
}

\section{Emerging 2D-material-based inks could find applications in next-generation printed electronics.}

Innovative technologies, however appealing, remain within the realm of academia until sufficiently mature. For businesses that generally run on narrow margins, the implementation of novel manufacturing processes is far too risky. Yet, every now and then, for the sake of marketing relevance and overall financial stability industries have to properly rethink the technological methods they are based upon. Even traditional printing, which comes across as a good example of technological longevity and adaptability, is currently at a crossroads with more people scrolling through their phone screens than flicking through the paper. In all-digital times, printed electronics may come to rescue and revolutionize the way we perceive modern print by imbuing paper with new functionality.

Modern printed electronics are an amalgam of traditional printing techniques, flexible substrates and solution-processable materials combined into one powerful technological platform. The promise of considerable manufacturing cost reduction, always at the forefront of technological evolution in industry, is again the main driver of scientific and technological interest in printed electronics. However, cost-effectiveness is not the only strong suit of this technology. Other potential advantages include faster production turnaround, lower operating cost and processing times, minimum maintenance, high material-utilization efficiency, use of flexible substrates and lightweight products, to name but a few. Besides, ambient fabrication favourably contrasts printing with traditional thin-film reliance on laborious high-temperature deposition. The portfolio of fabrication techniques for printed electronics comprises a wide range of methods. Some of them have already been applied to conventional electronics fabrication - including screen, inkjet, gravure, flexographic and offset printing.

Thus far, however, printed electronics hasn't been able to make its way from niche to mainstream market and is still described as novel, promising and emerging. It may come as a surprise to learn that patterning electronic components by printing is by no means a new idea. In fact, it's been nearly sixty years since printed circuits were first made commercially available. Back then, a simple brush was used to paint electrodes and resistive elements of basic electronic devices mounted onto ceramic plates. First, conducting paints were prepared in much the same way as it is done today from conductive powders mixed with binders to hold the particles together and solvents to ensure optimal fluidic properties. Finding the right balance between printability and functionality with limited choice of suitable materials has always been one of the biggest challenges for printed electronics. But with the recent advances in material science, this ultimate goal may finally be within reach.

The discovery of conducting polymers with inherent flexibility resulted in a sudden spurt in research activity towards applications in flexible electronics. Organic inks seem to be well suited to applications in wearable and consumer electronics, packaging, memory and energy storage. To that end, different research groups successfully demonstrated the fabrication of potentially scalable printed organic sensors, flexible displays, solar cells, luminescent films and smart labels. However, the lack of long-range order and stability of organics limits practically achievable mobility, which presents a serious challenge for most electronic devices. On the other hand, the use of high-mobility inorganic materials is hindered by scalability and integration issues.

The rise of graphene followed by isolation of other 2D materials such as black phosphorus, transition metal dichalcogenides (TMDs) and hexagonal boron nitride (h-BN) may foreshadow the next generation of printed electronics. These mechanically robust yet sufficiently flexible ultrathin materials can potentially fulfil the whole range of functionalities from conductors to insulators. What's more, a variety of heterojunctions can be formed by stacking individual layers into fundamental building blocks in different electronic devices. However, in the context of printing, exfoliated nanosheets have to be made into printable inks, ideally without any loss of their intrinsic properties. The current limitation comes from the use of solvents necessary to formulate stable dispersions with the right fluidic properties. Those that work best usually have high boiling points and are generally not environmentally benign. Using non-toxic solvents requires the addition of surfactants and binders, which usually support much higher loading, but can deteriorate the ink's properties. Despite many technical challenges, a number of graphene-based inks suitable for a variety of printing techniques, from inkjet to large-scale, high-speed, commercial printing have been successfully demonstrated. If this trend continues, graphene inks could soon become a viable alternative to inexpensive, poorly conducting carbon and more expensive, non-transparent silver currently used to fabricate conducting structures in many printed electronic devices. In this issue Daryl McManus and colleagues (page 343) take one step further towards printable $2 \mathrm{D}$ materials by developing new water-based and high-concentration inks formulated for materials including conductors (graphene), semiconductors $\left(\mathrm{MoS}_{2}, \mathrm{WS}_{2}\right)$ and insulators (h-BN). Moreover, these printable materials are potentially adaptable to biomedical applications owing to their experimentally confirmed biocompatibility. With this ink formulation method in hand, a variety of fully printed heterostructures can be realized directly onto plastic and paper substrates. Photosensors and programmable logic devices can be fabricated from inkjetprinted multi-stacks made of well-defined individual graphene and $\mathrm{WS}_{2}$ layers. The next challenge to overcome is the realization of printed thin-film transistors and, eventually, flexible circuits with superior characteristics than organic field-effect transistors.

All these recent advances open up new applications for low-dimensional materials, but most importantly, they can help in exploring and exploiting many benefits of printed electronics technology with the hope of extending its current applications beyond smart packaging and disposable sensors. As is often the case with printed electronics, it is yet to be seen whether the research efforts towards the development of printable 2D materials will have any far-reaching technological implications in the foreseeable future. Luckily, research has the luxury of going by trial-and-error, while industry can just sit and wait for the ripe fruits of all the scientific excitement surrounding $2 \mathrm{D}$ materials and their potential use in printed electronics.

\section{Correction}

In the Editorial 'Of knowledge and beauty' (Nat. Nanotech. 12, 185; 2017) the quote from Robert Boyle was misdated. The correct date is 1664. This was corrected after print in the online versions of the Editorial on 13 March 2017. 